\title{
Role of iron supplementation in promoting maternal and fetal outcome
}

This article was published in the following Dove Press journal:

Therapeutics and Clinical Risk Management

28 October 2011

Number of times this article has been viewed

\section{Zahra Yekta' \\ Reza Pourali² \\ Nikol Mladkova ${ }^{3}$ \\ Mohammad Ghasemi-rad ${ }^{4}$ \\ Farzane Boromand ${ }^{5}$ \\ Khosrow Hazrati Tappeh ${ }^{6}$ \\ 'Department of Community Medicine; ${ }^{2}$ Medical Demonstration Facility, Faculty of Medicine, Urmia University of Medical Sciences, Urmia, Islamic Republic of Iran; ${ }^{3}$ Institute of Cell and Molecular Science, London, United Kingdom; ${ }^{4}$ Student Research Committee; ${ }^{5}$ Department of Obstetrics and Gynecology; ${ }^{6}$ Department of Mycology and Parasitology, Urmia University of Medical Sciences, Urmia, Islamic Republic of Iran}

Correspondence: Mohammad Ghasemi-rad Student Research Committee, Urmia University of Medical Sciences,

Djahad St, PO Box II 38, Urmia, Islamic Republic of Iran

Tel +98 9l 43435284

Fax +98 44 I2353 356।

Email medmanII@gmail.com
Background: The data comparing daily versus intermittent iron supplementation during pregnancy remain controversial. This study was undertaken to compare the efficacy of daily versus two different intermittent iron supplementation regimes on hematologic markers and birth outcomes in nonanemic pregnant women.

Methods: Two hundred and ten women with singleton pregnancies, no known disease, and hemoglobin levels $>11.0 \mathrm{~g} / \mathrm{dL}$ were randomly assigned to one of three groups, ie, Group A consuming two iron supplementation tablets once weekly (100 mg iron per week, $\mathrm{n}=70$ ), Group B consuming one tablet twice weekly (100 mg iron per week, $\mathrm{n}=70)$ and Group C, consuming one tablet daily ( $50 \mathrm{mg}$ iron per day, $\mathrm{n}=70$ ). No additional micronutrients were supplied. Hemoglobin and serum ferritin levels were measured at 20, 28, and 38 weeks. Pregnancy and birth outcomes (pregnancy termination, method of delivery, birth weight, stillbirth) were analyzed.

Results: In total, 201 women completed the protocol. There was a significant difference in mean hemoglobin and ferritin levels in Group B at 38 weeks ( $P=0.018$ and $P=0.035$, respectively) but this difference was not clinically significant (hemoglobin $>12 \mathrm{~g} / \mathrm{dL}$, ferritin $>19 \mu \mathrm{g} / \mathrm{L}$ ). There was a significant increase in ferritin in Group $\mathrm{C}(P=0.03)$ at 28 weeks. No significant difference was observed with respect to pregnancy or birth outcome across the groups. All regimens prevented the occurrence of hemoglobin $<10.5 \mathrm{~g} / \mathrm{dL}$, but weekly supplementation was associated with development of a hemoglobin level $<11.0 \mathrm{~g} / \mathrm{dL}$ (risk ratio 0.044).

Conclusion: Twice-weekly supplementation is as effective as daily supplementation, and may represent an acceptable compromise in iron supplementation regimens for nonanemic pregnant women.

Keywords: iron supplementation, pregnancy, anemia, outcome

\section{Introduction}

Iron deficiency is considered to be the most prevalent nutrient deficiency in the world, and iron deficiency anemia is estimated to affect $1-2$ billion people globally. ${ }^{1}$ Groups demonstrating the highest prevalence of iron deficiency anemia are infants, children, and pregnant women. ${ }^{2}$ More than $50 \%$ of pregnant women in developing countries ${ }^{3}$ and approximately $20 \%$ in industrialized countries ${ }^{4}$ suffer from iron deficiency.

During pregnancy, iron is a critical element for both the pregnant mother and the growing fetus, ${ }^{5}$ and is essential to neurotransmitter synthesis and myelination of the central nervous system. Iron also functions as a cofactor for enzymes involved in brain development. ${ }^{6}$ Fetal iron supply is provided by deflection of a significant proportion of maternal iron to the developing fetus. ${ }^{7}$ 
Despite some controversy, anemia in pregnancy has been linked with preterm delivery and low birth weight, ${ }^{8}$ and maternal iron deficiency in pregnancy has been linked with altered neurocognitive development of the infant. ${ }^{3}$ Thus, determination of the potentially most effective approach towards prevention and accurate management of anemia in pregnancy represents a clinical problem with global impact.

Although the World Health Organization (WHO) published recommendations on the design of large-scale iron supplementation programs in $1989^{9}$ to alleviate this nutritional disease, iron supplementation during pregnancy remains a controversial issue. The ultimate goal of providing iron supplementation to a healthy, nonanemic pregnant woman is to ensure sufficient iron storage in her body to provide effective cover for the increased physiologic need of iron during pregnancy, because the natural mechanisms of increasing iron intake, such as higher absorption of iron from the gastrointestinal tract, are not always adequate. Women with iron deficiency are particularly susceptible to iron deficiency anemia during pregnancy, because the demand for iron increases from 0.8 to $\leq 7.5 \mathrm{mg}$ of absorbed iron per day. ${ }^{10}$ Latent or overt iron deficiency will gradually progress to iron deficiency anemia, characterized by microcytosis and increased amounts of protoporphyrin IX in red blood cells, ${ }^{4}$ which is linked with a risk of an unfavorable pregnancy outcome, as mentioned previously.

In the Islamic Republic of Iran, the prevalence of iron deficiency anemia in pregnant women varies between $2.4 \%$ and $27.8 \%$ with respect to specific regions, ${ }^{11}$ and thus represents a severe public health issue. The current policy of the Iranian Ministry of Health is to examine pregnant women for anemia at 12-17 weeks' gestation and to supply $30 \mathrm{mg} /$ day of iron as ferrous sulfate to each pregnant woman from gestational week 20 until three months after delivery. ${ }^{11}$

However, discussion regarding the true desirability of daily iron supplements versus intermittent iron supplements has arisen in recent years. The reasons for initial consideration of other than daily iron supplementation regimens are based on the lack of effectiveness of daily iron intervention in developing countries, ${ }^{12}$ indicated by low compliance with many daily supplementation programs, ${ }^{12}$ insufficient supply of iron, ${ }^{11}$ and insufficient coverage of at-risk populations. ${ }^{12}$ More evidence-based theory behind intermittent iron supplementation reflects the original concept of mucosal block of iron absorption due to an increase in the proportion of iron that is stored in the enterocyte, and a decrease in transfer of iron to transferrin in the vascular pool, ${ }^{13}$ confirmed decades later to some extent by in vivo studies in rats showing a rapid decline in the absorptive and iron retention capacities during daily iron administration compared with an intermittent regimen. Hence, daily iron supplementation may not be the most efficient approach towards maintaining or improving the iron status of a woman during pregnancy. ${ }^{14,15}$

This paper reports a randomized, unsupervised field study designed to evaluate the relative effectiveness of onceweekly, twice-weekly, and daily antenatal iron supplementation for maintaining safe hemoglobin and serum ferritin (indicator of iron reserves) levels during pregnancy and at delivery.

\section{Subjects and methods}

The study was conducted in North-West Iran in the urban regions of Urmia city. The study population was randomly drawn from pregnant women attending local public health care centers at seven prenatal health care clinics between September, 2007 and February, 2009. Thirty women were finally enrolled at each participating clinic. The women were invited to participate in the study during their first visit to the government antenatal clinic at 17-20 weeks and were informed by an assigned health care provider of its purpose, the procedures and tests entailed, and what data would be collected for analysis. Participants were free to withdraw from the study at any time without any disadvantage to themselves. Written informed consent was obtained from each literate woman and an improvised linear shaped signature in front of a witness was obtained on behalf of illiterate women who agreed to participate in the study. Before the start of the study, its protocol was approved by the University Ethics Committee and by the Faculty of Medicine. The protocol required participants to take the supplements on an assigned basis, to fill out forms about pill-taking and return these at each return clinic visit, and to have a $5 \mathrm{~mL}$ venipuncture for hemoglobin and ferritin concentration at 20,28, and 38 weeks' gestation. Women with previously demonstrated low hemoglobin levels or overt anemia were not eligible to participate. Exclusion criteria were hemoglobin $<11 \mathrm{~g} / \mathrm{dL}$, noncompliance, intolerance of iron tablets or a physician's recommendation to refrain from iron intake, previous consumption of iron-containing supplements, multiple pregnancy, thalassemia, chronic systemic disease, eclampsia or pre-eclampsia, more than five previous completed pregnancies, history of bleeding or surgery during previous pregnancy, pregnancy terminated before 36 weeks, and relocation to another area. 


\section{Sample size, allocation of group, and tablet intake}

A total of 210 nonanemic pregnant women with a hemoglobin level $>11.0 \mathrm{~g} / \mathrm{dL}$, set as a cutoff value for anemia based on the WHO definition, ${ }^{16}$ were recruited at $>17$ and $<20$ weeks of gestation for participation in this study. The power of the study was $80 \%$, and the level of significance was considered to be $P<0.05$. The prevalence of anemia at delivery in the daily control group versus weekly group was reported to be $9 \%$ versus $45 \%$, respectively, according to a literature review. The women were randomly assigned to receive two iron tablets once weekly $(100 \mathrm{mg} / \mathrm{dose}$, Group A), one tablet twice weekly (50 mg/dose, Group B), or once daily (50 mg/day) as ferrous sulfate. The weekly supplemented group received two $50 \mathrm{mg}$ ferrous sulfate tablets on Friday, and the twice-weekly supplemented group received one $50 \mathrm{mg}$ ferrous sulfate tablet on Monday and Wednesday. Therefore, both groups received the same total amount of elemental iron per week, ie, $100 \mathrm{mg}$. All women were instructed to take the tablets on an empty stomach before sleep.

Subsequently, at 28 weeks' gestation, four women were excluded due to hemoglobin $<11 \mathrm{~g} / \mathrm{dL}$, lack of compliance $(n=2$, Groups A and B) or relocation to another area $(\mathrm{n}=2$, Group A). Two hundred and six women continued to participate in the study beyond 28 weeks. Data for the excluded women were not included in the final analysis.

At 38 weeks, three women from Group A were excluded due to hemoglobin levels $<11 \mathrm{~g} / \mathrm{dL}$ and two women decided of their own volition to transfer to a daily supplementation regimen based on the recommendation of their private physician, in spite of having safe hemoglobin levels $(12.8 \mathrm{~g} / \mathrm{dL}$ and $11.7 \mathrm{~g} / \mathrm{dL}$ ). Two hundred and one women completed the study protocol and their data were analyzed. The study outline is summarized in Figure 1.

Data on age, body weight, parity, and height were recorded at the time of study enrolment (Table 1). Body mass index was calculated according to the standard formula as weight in kilograms divided by the square of height in meters, and weight gain was assessed by the difference in weight at 20 and 38 weeks' gestation. Parity was determined by self-reporting and calculated as the number of deliveries assigned to a particular group divided by total number of completed pregnancies reported by women in the group.

\section{Serum ferritin and hemoglobin levels}

Participants were sent to a reference laboratory at assigned weeks of gestation according to the protocol to provide venous blood samples for assessment of hemoglobin and serum

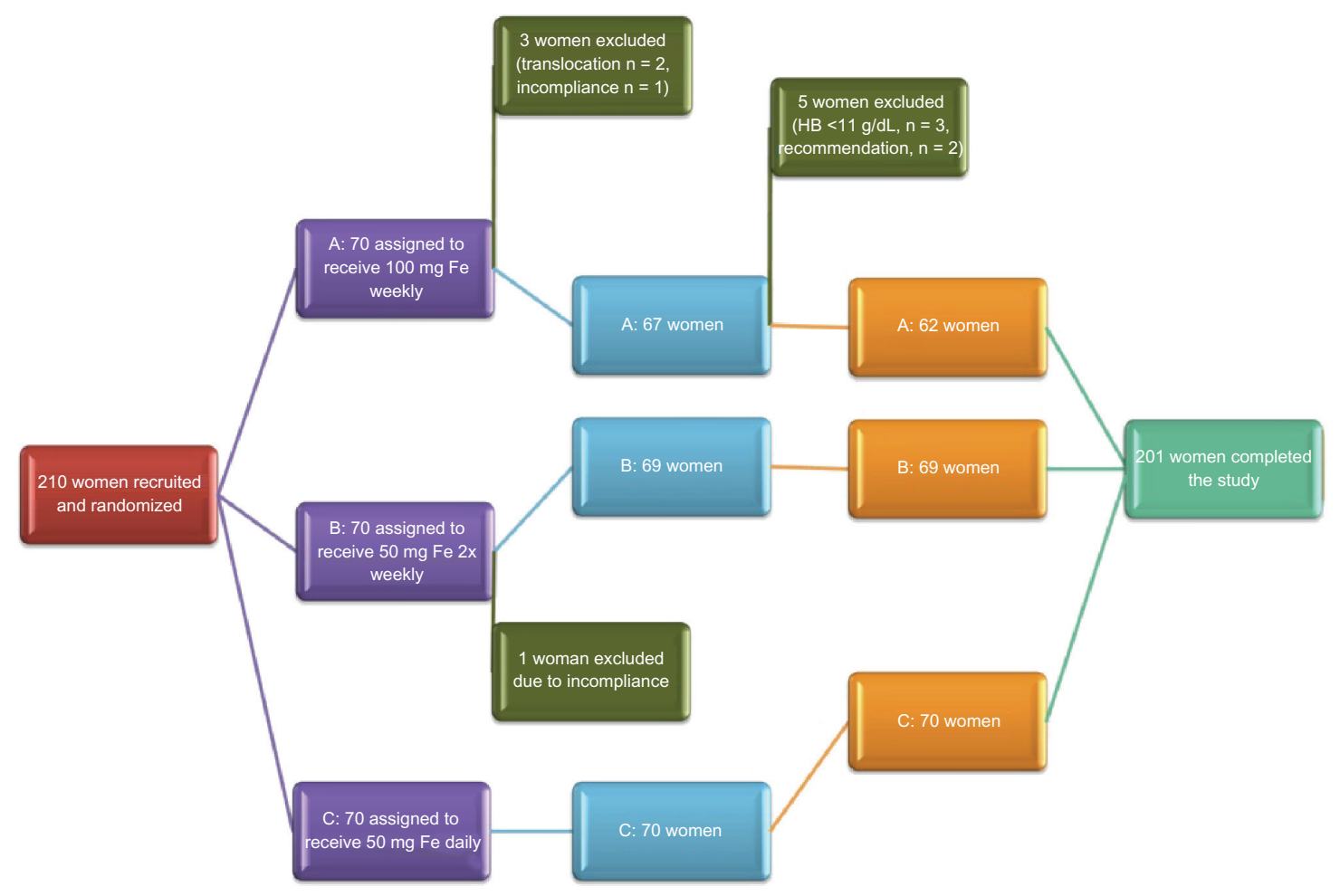

Figure I Overview of clinical study. 
Table I Geometric mean ( \pm standard deviation) of baseline characteristics of participating women at time of enrolment $(\leq 20$ weeks' gestation) and delivery

\begin{tabular}{llllll}
\hline Weeks of gestation & Characteristic & Group A & Group B & Group C & P value \\
\hline$\leq 20$ & Age \pm SD & $25.8 \pm 4.9$ & $24.3 \pm 4.6$ & $25.4 \pm 5.4$ & 0.21 \\
& BMI (I7-19 weeks) \pm SD & $26.3 \pm 4$ & $26.1 \pm 4.8$ & $25.8 \pm 4.3$ & 0.75 \\
& Parity & 0.76 & 0.65 & 0.77 & 0.5 \\
& Nulliparity & $33(53.2 \%)$ & $38(55.1 \%)$ & $33(47.1 \%)$ & \\
38 & & & & $3.4 \pm 1.5$ & 0.61 \\
& Weight gain (20-38 weeks) \pm SD & $3.2 \pm 3.5$ & $3.1 \pm 1.3$ & $29.2 \pm 4.3$ & 0.61 \\
\hline
\end{tabular}

Abbreviations: BMI, body mass index; SD, standard deviation.

ferritin concentrations. Women with hemoglobin $<11 \mathrm{~g} / \mathrm{dL}$ at or after 28 weeks' gestation ( $\mathrm{n}=3$, Group A) were excluded from the study and referred to a physician to be treated with daily supplementation of two $50 \mathrm{mg}$ ferrous sulfate tablets until the end of their pregnancy, and this supplementation regimen was continued for three months after delivery. The remaining participants were followed until delivery and a blood sample was obtained according to the protocol at 38 weeks' gestation. After delivery, data on infant outcome (birth weight and gestational age) were collected from the family medical file at the local health center and analyzed. Venous blood samples of $5 \mathrm{~mL}$ obtained at 20, 28, and 38 weeks' gestation were analyzed for hemoglobin level by the antenatal clinic reference laboratory using an $\mathrm{H} 1$ automated counter (Technicon, Bayer, Tehran, Iran). At these times, a second tube of venous blood was collected for measurement of serum ferritin concentration and kept refrigerated until analysis. Samples for that assessment were analysed in the same laboratory using an immunoradiometric assay technique (Kavoshyar Ferritin IRMA $\left[\mathrm{I}_{125}\right]$ kit, Kavoshyar Iran Co, Tehran, Iran).

\section{Adherence to regimen and side effects}

Overall adherence to the regime was calculated as the number of tablets women swallowed according to given forms, divided by the total number of tablets they should have received during supplementation, ie, number of weeks enrolled in the program multiplied by the number of tablets received in each group. Side effects were recorded at regular visits.

\section{Medical record extraction}

Birth weight and date of delivery were extracted by health care providers from the family file using standardized data entry forms for all women who completed the study protocol and who delivered at the local medical center at Koversusar Hospital, Urmia. Each women who delivers in a government center is given a form which she brings to the hospital and fills out after the birth before being discharged, which is then maintained in a family file.

\section{Birth outcomes}

Low birth weight was defined as $<2500 \mathrm{~g}$. Gestational age was determined either on the basis of a participant-reported estimate of the last menstrual period or by an ultrasound examination performed early in the pregnancy. Determination of preterm birth was based solely on duration of gestation, with deliveries that occurred at $<37$ completed weeks designated as preterm. Deliveries via cesarean section and stillbirths were noted.

\section{Statistical analysis}

An analysis of variance test was used to assess baseline characteristics across the groups. We conducted analyses on outcomes based on randomization of the study participants and compared the average measurements at the designated time points by randomization group. Kruskal-Wallis nonparametric analysis of variance was used for comparison of mean ferritin and hemoglobin levels across the groups. Unpaired $t$-tests were used to compare continuous outcomes between the randomization groups, and Chi-squared tests and post hoc tests, where appropriate, were used to compare differences in categorical outcomes. Risk ratios and 95\% confidence intervals were estimated for comparisons between the supplementation groups for the outcomes of preterm delivery. Statistical significance was set at $P<0.05$. The Statistical Package for Social Sciences for Windows version 16.0 (SPSS Inc, Chicago, IL) was used for all analyses.

\section{Results}

\section{Characteristics of study sample and participation}

A total of 210 women were initially invited to participate in the study and randomly assigned to one of three treatment groups, ie, 70 in Group A, 70 in Group B, and 70 in Group C. 
Of these, four $(1.9 \%)$ were excluded at 28 weeks and five $(2.38 \%)$ at 38 weeks due to detection of exclusion criteria, leading to a total dropout rate of $4.28 \%$. Two hundred and one remaining subjects (95.72\%; 62 in Group A, 69 in Group $\mathrm{B}$, and 70 in Group C) completed the study protocol, and had blood samples taken at baseline and at all assigned time points after supplementation (Figure 2). The participants were matched according to body mass index $(P=0.75)$, parity $(P=0.50)$, age $(P=0.21)$, baseline hemoglobin $(P=0.7)$, and ferritin levels $(P=0.37)$.

Withdrawals from the study before 36 weeks' gestation were due to relocation ( $\mathrm{n}=2$, Group A) noncompliance ( $\mathrm{n}=2$, Group A and Group B), and hemoglobin $<11 \mathrm{~g} / \mathrm{dL}$ at 38 weeks ( $n=3$, Group A) and were therefore excluded from the study and treated appropriately. Two women withdrew on the recommendation of their physician.

The baseline characteristics of the participating women by treatment group are shown in Table 1. The women did not differ in any of the physiologic study variables (body mass index, age, parity, weight gain), or initial hemoglobin or ferritin concentrations.

The mean age of the participating women was $25.5 \pm 5.04$ (range 19.7-30.7) years; $49.2 \%$ of the participants were aged $19-24$ years, $27.7 \%$ were aged $25-29$ years, and $23.1 \%$ were aged 30 years or older. More than $50 \%$ of participants $(\mathrm{n}=104)$ were nulliparous at study entry. The average baseline hemoglobin and ferritin concentrations were $12.03 \pm 0.79 \mathrm{~g} / \mathrm{dL}$ and $14.16 \pm 11.13 \mu \mathrm{g} / \mathrm{L}$, respectively. The baseline range for hemoglobin concentration was $11.19-12.93 \mathrm{~g} / \mathrm{dL}$.

\section{Iron status and relative effectiveness of iron supplementation regimens}

Table 2 summarizes the data comparing mean hemoglobin and serum ferritin levels for women who completed the study as measured at designated time points. Figures 2 and 3 show time point-correlated geometric mean values for hemoglobin.

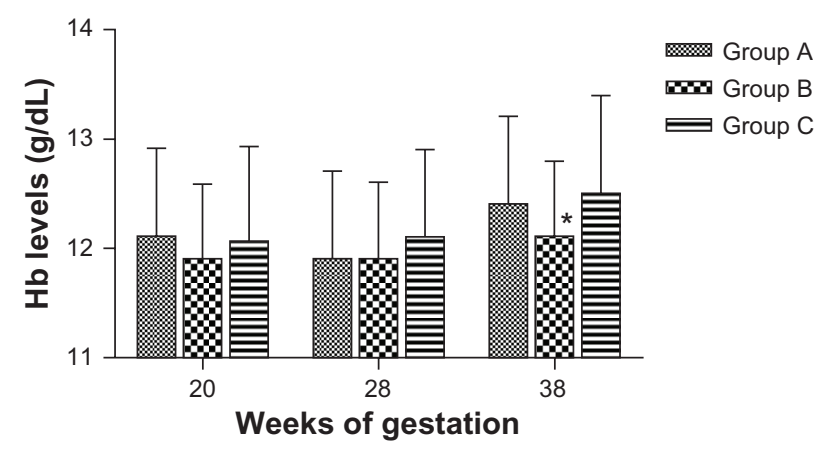

Figure 2 Geometric means of hemoglobin level. Note: *Statistically significant.
No significant difference was found between the treatment groups for hemoglobin levels at 28 weeks. At 38 weeks, the mean hemoglobin level in Group B was significantly lower than in Group A and Group C $(P=0.018)$, but the difference was of no clinical significance. The mean serum ferritin level increased significantly in Group C at 28 weeks' gestation $(P=0.03)$. When comparing the final levels at 38 weeks, a significant difference was observed between Group B and the other two groups in this iron status indicator $(P=0.035)$. This difference was also of marginal clinical significance (19.9 $\mu \mathrm{g} / \mathrm{L}$ in Group B).

\section{Birth outcome}

An overall comparison of average birth parameters for the three treatment groups is summarized in Table 3. Infants born to women who were assigned to the different treatment groups showed no significant difference in average birth weight $(P=0.521)$. The difference in pregnancy termination week was insignificant across the randomization groups $(P=0.13)$, as was the incidence of cesarean section births, preterm delivery, and low birth weight across the groups ( $P=0.31,0.25$, and 0.33 , respectively). Two babies (one in Group A and one in Group C) were of low birth weight, and eight babies (Group A, n= 4; Group B, n= 3; Group C, $\mathrm{n}=1$ ) were delivered preterm.

\section{Adherence to regimen and side effects}

Adherence to the regime was $100 \%$ according to data provided in the forms for the participants. No side effects were reported throughout the duration of the study.

\section{Discussion}

Low hemoglobin caused most commonly by iron deficiency is highly prevalent in pregnant women, and represents a global clinical problem with considerable impact on public health and health care systems. ${ }^{1}$ Various routine iron supplementation regimes have been proposed in recent decades to address this issue from the public health perspective, most commonly consisting of daily iron supplementation. Our data suggest that there is no significant benefit of daily iron supplementation over intermittent dosing regimes for maintenance of safe hematologic marker levels and for preventing pregnancy and neonatal complications in pregnant women who are nonanemic at $\leq 20$ weeks. The absence of a placebo control group allows only a comparison between daily, twice-weekly, and once-weekly supplementation groups. However, given that it has been well established by various studies that daily antenatal iron supplementation increases hemoglobin levels 
Table 2 Effect of once-weekly, twice-weekly, and daily iron supplementation on hemoglobin and ferritin levels in nonanemic pregnant women

\begin{tabular}{|c|c|c|c|c|c|c|}
\hline \multirow{2}{*}{$\begin{array}{l}\text { Weeks of } \\
\text { gestation }\end{array}$} & \multicolumn{2}{|c|}{ Group A $(n=62)$} & \multicolumn{2}{|c|}{ Group B $(n=69)$} & \multicolumn{2}{|c|}{ Group C $(n=70)$} \\
\hline & Hemoglobin & Ferritin & Hemoglobin & Ferritin & Hemoglobin & Ferritin \\
\hline 20 & $|2.1 \pm 0.8|$ & $13.4 \pm 9.6$ & $11.9 \pm 0.69$ & $13.5 \pm 10.1$ & $12.06 \pm 0.87$ & $15.8 \pm 13$ \\
\hline 28 & $11.9 \pm 0.82$ & $16.09 \pm 9.8$ & $11.9 \pm 0.75$ & $14.2 \pm 9.1$ & $12.1 \pm 0.83$ & $18.8 \pm 12.1^{*}$ \\
\hline 38 & $12.4 \pm 0.85$ & $21.3 \pm 11.07$ & $12.1 \pm 0.78^{*}$ & $19.9 \pm 11.2^{*}$ & $12.5 \pm 0.93$ & $25.3 \pm 13.2$ \\
\hline
\end{tabular}

Note: *Statistically significant.

in maternal blood both antenatally and postnatally, ${ }^{17}$ that absence of iron supplementation during pregnancy increases the risk of anemia, ${ }^{18}$ and that various countries now recommend routine iron supplementation as a conceptual part of their public health care policy (US, France, ${ }^{19}$ Iran), our goal was not to assess the impact of absence of iron supplementation in comparison with iron supplies, but to compare the effect of various iron supplementation regimens in a matched sample of pregnant women.

In the women who were nonanemic at $20 \leq$ weeks' gestation, weekly supplementation was less effective in preventing hemoglobin levels $<11 \mathrm{~g} / \mathrm{dL}$ compared with twice-weekly and daily supplementation regimes. However, the twice-weekly regime has shown equal effectiveness as a daily supplementation regimen with respect to maintaining safe hemoglobin and ferritin levels throughout pregnancy. In addition to this, based on WHO criteria for the lowest (5th percentile) mid-pregnancy hemoglobin level of $10.5 \mathrm{~g} / \mathrm{dL},{ }^{16}$ also proposed by Milman et al, ${ }^{20}$ none of the women who were excluded due to hemoglobin levels $<11 \mathrm{~g} / \mathrm{dL}$ at 28 weeks were in fact anemic $(10.6,10.7$, and $10.7 \mathrm{~g} / \mathrm{dL})$. Moreover, this finding could be explained by the physiologic hemodilution that occurs during this stage of pregnancy. The reason they were transferred to a daily supplementation regimen was based on a cutoff point of $11 \mathrm{~g} / \mathrm{dL}$ for anemia in pregnant women throughout pregnancy applied in routine Iranian

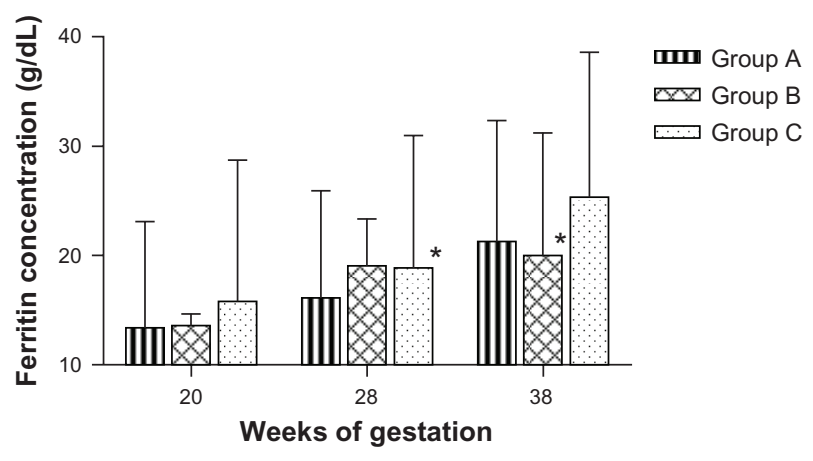

Figure 3 Geometric means of ferritin level. Note: *statistically significant. clinical practice in accordance with the Iranian Ministry of Health recommendation.

Although hemoglobin levels were significantly lower in Group B compared with Groups A and C $(P=0.018)$ at 38 weeks, the difference was not clinically significant $(\mathrm{Hb}$ level $>11 \mathrm{~g} / \mathrm{dL}$ ). A similar trend was observed for the difference in ferritin levels, in that although daily supplementation led to a significant increase in ferritin at 28 weeks $(P=0.03)$, this difference was not maintained towards 38 weeks in comparison with the other two regimes. Group B, on the other hand, achieved significantly lower ferritin levels compared with Group A and Group C at 38 weeks, but again this was not clinically significant.

Various studies comparing daily versus intermittent regimens have shown that the intermittent regimen is less effective in anemic pregnant women, ${ }^{21,22}$ while other studies conducted in anemic ${ }^{23}$ and nonanemic ${ }^{24}$ women demonstrate equal efficacy, ${ }^{25}$ frequently due to poor adherence to the regime in the daily supplementation setting. In addition to equal ability to maintain safe hematologic markers on both regimes, studies also report a possible adverse effect of hemoconcentration on birth outcome ${ }^{24}$ and other aspects associated with daily exposure to iron, such as hypertension, in healthy nonanemic women. ${ }^{26}$

At 38 weeks, $15.7 \%$ women in Group $\mathrm{C}$ had hemoconcentration, compared with $8.1 \%$ and $7.3 \%$ in Group A and Group B, respectively. However, the initial hemoglobin levels were higher in Group C (12.9\% had hemoconcentration at week 20 compared with 5.3\% in Group B), which may account for the considerably higher percentage of women with hemoconcentration in this group at the time of delivery.

It is noteworthy that many studies comparing daily versus intermittent iron supplementation attribute the low effectiveness of routine daily administration to low adherence with the daily regimen, ${ }^{21,27}$ which is mainly due to the side effects of daily iron supplementation. Given the fact that all women in our study reported full adherence and no side effects, it may be important to study the social characteristics of target study populations to verify this finding. 
Table 3 Pregnancy outcome analysis in randomization groups

\begin{tabular}{|c|c|c|c|c|}
\hline Variable & Group A & Group B & Group C & $P$ value \\
\hline Birth weight & $3212 \pm 461.4$ & $3222.38 \pm 500$ & $3274.26 \pm 408.3$ & $0.7 \mathrm{~F}=0.35$ \\
\hline Pregnancy termination week & $39.9 \pm 1.2$ & $39.5 \pm 1.4$ & $40.03 \pm 1.6$ & $0.12 \mathrm{~F}=2.1$ \\
\hline $\mathrm{C} / \mathrm{S}$ & 14 (20.9\%) & $22(31.9 \%)$ & 21 (30.9\%) & 0.29 \\
\hline Preterm delivery & $4(7.5 \%)$ & $3(5.8 \%)$ & I (1.4\%) & 0.25 \\
\hline LBW & $\mathrm{I}(\mathrm{I} .5 \%)$ & $0(0 \%)$ & $\mathrm{I}(\mathrm{I} .4 \%)$ & 0.61 \\
\hline
\end{tabular}

Abbreviations: LBW, low birth weight; C/S, cesarean section.

Another aspect that may have played a role in the significantly high adherence during this study is that all women were informed in detail about the general effects and benefits of iron on the course of their pregnancy and its outcome, and were clearly instructed on how and when to take their tablet(s). This is in accordance with another compliance study demonstrating that this approach increases compliance rates. ${ }^{27}$

The dietary habits of the study sample were not specifically recorded, but given the relative ethnic, religious, and socioeconomic homogeneity of the participating women, it may be reasonably assumed that there were no significant differences in their diet. None of the participating women were vegetarians, and all were informed regarding proper dietary composition during pregnancy. In accordance with Islamic law, which does not allow pregnant women to fast, none were fasting. Several studies have demonstrated that anemia in pregnancy is associated with a risk of low birth weight or premature delivery. ${ }^{28-30}$ Our study shows that low birth weight occurred with equal frequency in the daily and weekly supplementation groups ( $\mathrm{n}=1$ in each group), while no low birth weight infants occurred in the twice-weekly group.

Pan-population approaches using global routine iron supplementation, although introduced, eg, in India in the early $70 \mathrm{~s},{ }^{21}$ have proven to be ineffective in reducing the prevalence of anemia during pregnancy. Moreover, such approaches may not be safe in all circumstances, as shown by studies demonstrating an adverse effect on pregnancy outcome in women with higher hemoglobin levels who receive iron supplementation. Currently there is no general solution for this worldwide problem, which rather emphasizes the need for careful evaluation of target populations with respect to social, dietary, and religious customs, and in particular the need for an individual approach in each pregnant woman. Rigid iron supplementation rules based on inaccurate generalizations from studies conducted in different global and political regions in different ethnic populations and sometimes in a different decade may eventually cause harm instead of benefit in a subset of pregnant women and their babies.
In conclusion, our study shows that assessment of hematologic parameters in early pregnancy should be routinely incorporated into prenatal care and, based on this evaluation, appropriate iron supplementation should be proposed for every woman, regardless of location or background. This regimen should be in accordance with local and national population health and the woman's individual medical history and needs. From a more general perspective, a heated debate concerning daily versus intermittent iron supplementation has developed in recent years. Both approaches have been corroborated with clinical trials, yet no clear conclusion can be reached. Although our results do not provide a definite answer, they do suggest that, in nonanemic women, a twice-weekly iron supplementation regime may represent a reasonable compromise for an initial iron dosing regimen in nonpregnant women that may be adjusted in concordance with the development of iron markers during pregnancy.

\section{Acknowledgment}

We would like to thank the Student Research Committee, Urmia University of Medical Sciences, for their support of this research.

\section{Disclosure}

The authors report no conflicts of interest in this work.

\section{Reference}

1. Stoltzfus R. Defining iron-deficiency anemia in public health terms: a time for reflection. $J$ Nutr. 2001;131(2S-2):565S-567S.

2. Sloan NL, Jordan E, Winikoff B. Effects of iron supplementation on maternal hematologic status in pregnancy. Am J Public Health 2002;92(2):288-293.

3. Lozoff B, Georgieff MK. Iron deficiency and brain development. Semin Pediatr Neurol. 2006;13(3):158-165.

4. Haram K, Nilsen ST, Ulvik RJ. Iron supplementation in pregnancy - evidence and controversies. Acta Obstet Gynecol Scand. 2001;80(8):683-688.

5. Milman N. Iron prophylaxis in pregnancy - general or individual and in which dose? Ann Hematol. 2006;85(12):821-828.

6. LeBlanc CP, Fiset S, Surette ME, Turgeon O'Brien H, Rioux FM. Maternal iron deficiency alters essential fatty acid and eicosanoid metabolism and increases locomotion in adult guinea pig offspring. JNutr. 2009;139(9):1653-1659. 
7. Andrews NC. Iron homeostasis: insights from genetics and animal models. Nat Rev Genet. 2000;1(3):208-217.

8. Hematologic disorders of pregnancy. 3rd ed. In: Fortner KB, Szymanski L, Fox HE, Wallach EE, editors. The Johns Hopkins Manual of Gynecology and Obstetrics. 3rd ed. Philadelphia, PA: Lippincott Williams and Wilkins; 2007:215-230.

9. DeMaeyer EM, Dallman P, Gurney JM, Hallberg L, Sood SK, Srikantia SG. Preventing and controlling iron deficiency anaemia through primary health care. Geneva: World Health Organization; 1989.

10. Beard JL. Effectiveness and strategies of iron supplementation during pregnancy. Am J Clin Nutr. 2000;71(5 Suppl):1288S-1294S.

11. Yekta Z, Ayatollahi H, Pourali R, Farzin A. Predicting factors in iron supplement intake among pregnant women in urban care setting. JRHS. 2008;8(1).

12. Beard JL. Weekly iron intervention: the case for intermittent iron supplementation. Am J Clin Nutr. 1998;68(2):209-212.

13. Hahn PF, Bale WF, Ross JF, Balfour WM, Whipple GH. Radioactive iron absorption by gastro-intestinal tract: influence of anemia, anoxia, and antecedent feeding distribution in growing dogs. J Exp Med. 1943;78(3):169-188.

14. Wright AJ, Southon S. The effectiveness of various iron-supplementation regimens in improving the Fe status of anaemic rats. Br J Nutr. 1990;63(3):579-585.

15. Viteri FE, Liu X, Tolomei K, Martin A. True absorption and retention of supplemental iron is more efficient when iron is administered every three days rather than daily to iron-normal and iron-deficient rats. J Nutr. 1995; 125(1):82-91.

16. Prevention and management of severe anaemia in pregnancy. Report of a Technical Working Group, Geneva, Switzerland: World Health Organisation; 1993.

17. Pena-Rosas JP, Viteri FE. Effects of routine oral iron supplementation with or without folic acid for women during pregnancy. Cochrane Database Syst Rev. 2006;3:CD004736.

18. Preziosi P, Prual A, Galan P, Daouda H, Boureima H, Hercberg S. Effect of iron supplementation on the iron status of pregnant women: consequences for newborns. Am J Clin Nutr. 1997;66(5):1178-1182.

19. Makrides M, Crowther CA, Gibson RA, Gibson RS, Skeaff CM. Efficacy and tolerability of low-dose iron supplements during pregnancy: a randomized controlled trial. Am J Clin Nutr. 2003;78(1):145-153.
20. Milman N, Byg KE, Agger AO. Hemoglobin and erythrocyte indices during normal pregnancy and postpartum in 206 women with and without iron supplementation. Acta Obstet Gynecol Scand. 2000;79(2):89-98.

21. Mukhopadhyay A, Bhatla N, Kriplani A, Pandey RM, Saxena R. Daily versus intermittent iron supplementation in pregnant women: hematological and pregnancy outcome. J Obstet Gynaecol Res. 2004;30(6):409-417.

22. Mumtaz Z, Shahab S, Butt N, Rab MA, DeMuynck A. Daily iron supplementation is more effective than twice weekly iron supplementation in pregnant women in Pakistan in a randomized double-blind clinical trial. J Nutr. 2000;130(11):2697-2702.

23. Young MW, Lupafya E, Kapenda E, Bobrow EA. The effectiveness of weekly iron supplementation in pregnant women of rural northern Malawi. Trop Doct. 2000;30(2):84-88.

24. Casanueva E, Viteri FE, Mares-Galindo M, et al. Weekly iron as a safe alternative to daily supplementation for nonanemic pregnant women. Arch Med Res. 2006;37(5):674-682.

25. Ridwan E, Schultink W, Dillon D, Gross R. Effects of weekly iron supplementation on pregnant Indonesian women are similar to those of daily supplementation. Am J Clin Nutr. 1996;63(6):884-890.

26. Ziaei S, Norrozi M, Faghihzadeh S, Jafarbegloo E. A randomised placebo-controlled trial to determine the effect of iron supplementation on pregnancy outcome in pregnant women with haemoglobin $>$ or $=13.2 \mathrm{~g} / \mathrm{dl}$. BJOG. 2007;114(6):684-688

27. Habib F, Alabdin EH, Alenazy M, Nooh R. Compliance to iron supplementation during pregnancy. J Obstet Gynaecol. 2009;29(6): 487-492.

28. Kidanto HL, Mogren I, Lindmark G, Massawe S, Nystrom L. Risks for preterm delivery and low birth weight are independently increased by severity of maternal anaemia. S Afr Med J. 2009;99(2):98-102.

29. Lone FW, Qureshi RN, Emmanuel F. Maternal anaemia and its impact on perinatal outcome in a tertiary care hospital in Pakistan. East Mediterr Health J. 2004;10(6):801-807.

30. Scanlon KS, Yip R, Schieve LA, Cogswell ME. High and low hemoglobin levels during pregnancy: differential risks for preterm birth and small for gestational age. Obstet Gynecol. 2000;96(5 Pt 1): 741-748.
Therapeutics and Clinical Risk Management

\section{Publish your work in this journal}

Therapeutics and Clinical Risk Management is an international, peerreviewed journal of clinical therapeutics and risk management, focusing on concise rapid reporting of clinical studies in all therapeutic areas outcomes, safety, and programs for the effective, safe, and sustained use of medicines. This journal is indexed on PubMed Central, CAS,

\section{Dovepress}

EMBase, Scopus and the Elsevier Bibliographic databases. The manuscript management system is completely online and includes a very quick and fair peer-review system, which is all easy to use. Visit http://www.dovepress.com/testimonials.php to read real quotes from published authors. 